\title{
Pattern of lymphatic spread in thoracic esophageal squamous cell carcinoma: A single-institution experience
}

\author{
Bin Li, MD, ${ }^{\text {a,b }}$ Haiquan Chen, MD, a,b Jiaqing Xiang, MD, ${ }^{\text {a,b }}$ Yawei Zhang, MD, ${ }^{\text {a,b }}$ Chenguang Li, MD, \\ Haichuan $\mathrm{Hu}, \mathrm{MD},{ }^{\mathrm{a}, \mathrm{b}}$ and Yang Zhang, $\mathrm{MD}^{\mathrm{a}, \mathrm{b}}$
}

\begin{abstract}
Objectives: Lymph node metastasis is among the most important prognostic factors for patients with esophageal squamous cell carcinoma after curative esophagectomy; however, the extent of lymphadenectomy is still controversial. The objective of the present study was to determine the frequency of lymphatic metastases and to study the pattern of lymph node metastasis in a large study population.
\end{abstract}

\begin{abstract}
Methods: The data from 1361 patients with thoracic esophageal squamous cell carcinoma who underwent curative $\mathrm{R} 0$ esophagectomy were retrospectively examined. Logistic regression analysis was used to identify the factors associated with lymph node metastasis.
\end{abstract}

Results: Of the 1361 patients, $714(52.5 \%)$ were found to have lymph node metastasis. The frequency of lymph node metastasis increased as the tumor invasion increased. Paratracheal nodes were the most frequent metastasis nodes $(15.9 \%)$. The frequency of lymph node metastasis was $9.8 \%$ in the neck, $18.0 \%$ in the upper mediastinum, $18.9 \%$ in the middle mediastinum, $11.8 \%$ in the lower mediastinum, and $28.4 \%$ in the abdomen. Of these 714 patients, $424(31.2 \%)$ presented with 1 field involvement, $255(18.7 \%)$ with 2 fields, and $35(2.6 \%)$ with 3 fields involvement. Logistic regression analysis revealed tumor length $(P<.001)$, tumor invasion $(P<.001)$, tumor differentiation $(P=.003)$, and lymphovascular invasion $(P<.001)$ were risk factors for lymph node metastasis. Tumor location $(P<.001)$, tumor invasion $(P=.003)$, lymphovascular invasion $(P=.004)$, and paratracheal lymph node involvement $(P=.002)$ were identified as risk factors for cervical lymph node metastasis.

Conclusions: Metastases were more frequent in the abdomen than in the neck. Total mediastinal and upper abdominal lymphadenectomy should be carefully conducted. Certain factors, such as tumor location, depth of tumor invasion, lymphovascular invasion, and paratracheal lymph node involvement, might be helpful in determining the need to perform cervical lymphadenectomy in individual patients. (J Thorac Cardiovasc Surg 2012;144:778-86)

Earn CME credits at

http://cme.ctsnetjournals.org

Lymph node metastasis (LNM) is one of the most important factors in predicting the prognosis of patients with esophageal carcinoma, ${ }^{1}$ but the optimal extent of lymphadenectomy is still debated. ${ }^{2,3}$ Studying the pattern of LNM might provide indications of when to perform lymph node dissection in individual patients. However, published studies of the pattern of lymphatic spread have differed owing to the

\footnotetext{
From the Department of Thoracic Surgery, ${ }^{a}$ Fudan University Shanghai Cancer Center, Shanghai, China; and Department of Oncology, ${ }^{\mathrm{b}}$ Shanghai Medical College, Fudan University, Shanghai, China

Disclosures: Authors have nothing to disclose with regard to commercial support.

Read at the 92nd Annual Meeting of The American Association for Thoracic Surgery, San Francisco, California, April 28-May 2, 2012.

Received for publication March 16, 2012; revisions received June 6, 2012; accepted for publication July 9, 2012; available ahead of print Aug 13, 2012.

Address for reprints: Haiquan Chen, MD, Department of Thoracic Surgery, Fudan University Shanghai Cancer Center, 270 Dong'an Rd, Shanghai 20032, China (E-mail: hqchen1@yahoo.com).

$0022-5223 / \$ 36.00$

Copyright (c) 2012 by The American Association for Thoracic Surgery

http://dx.doi.org/10.1016/j.jtcvs.2012.07.002
}

different treatment strategies used., ${ }^{4,5}$ Therefore, we reviewed our experience, including all the patients who had undergone radical lymphadenectomy at Fudan University Shanghai Cancer Center from 2006 to 2010. The goal of the present study was to accurately clarify the distribution of LNMs in thoracic esophageal squamous cell carcinoma (ESCC), and identify the factors related to LNM.

\section{PATIENTS AND METHODS \\ Patients}

The institutional review board of Fudan University Shanghai Cancer Center approved the database of esophageal carcinoma used for the present study. From January 2006 to December 2010, 1562 patients underwent esophagectomy at Fudan University Shanghai Cancer Center. The records of all patients with ESCC were reviewed for the present study. Of these patients, those who underwent transhiatal esophagectomy and received chemotherapy or radiotherapy before surgery were excluded. In addition, patients with fewer than 6 lymph nodes removed were excluded to minimize the potential for understaging. Thus, 1361 patients with ESCC who had undergone curative R0 transthoracic esophagectomy were included in the present retrospective study (Figure 1). Of the 1361 patients, 1107 were men $(81.3 \%)$ and 254 were women $(18.7 \%)$, with a mean age of $59 \pm 8$ years. A total of 294 patients $(21.6 \%)$ underwent 3-field lymphadenectomy (3-FL) and 1067 (78.4\%) 2-FL (Table 1). All patients were staged according to the TNM classification of the 7 th edition of the American Joint Committee for Caner staging manuals. ${ }^{6}$ The 


\section{Abbreviations and Acronyms}

2-FL $=2$-field lymphadenectomy

3-FL $=3$-field lymphadenectomy

$\mathrm{CT}=$ computed tomography

$\mathrm{ESCC}=$ esophageal squamous cell carcinoma

$\mathrm{LNM}=$ lymph node metastasis

pathologic stage was stage 0 in $12(0.9 \%)$, stage IA in $23(1.7 \%)$, stage IB in $115(8.4 \%)$, stage IIA in $186(13.7 \%)$, stage IIB in $437(32.1 \%)$, stage IIIA in $312(22.9 \%)$, stage IIIB in $153(11.2 \%)$, and stage IIIC in $123(9.0 \%)$ patients.

\section{Preoperative Workup}

According to the practice guidelines of the Shanghai Cancer Center, the preoperative workup included a complete history, physical examination, endoscopy of the upper gastrointestinal tract, computed tomography (CT) of the chest, and ultrasonography/CT of the neck and abdomen. Histologic confirmation of the carcinoma by endoscopy was also required. Positron emission tomography/CT and endoscopic ultrasonography were not routinely performed. On the basis of the results from these examinations, the patients who were medically suitable, with stage T1-T3 tumors without distant metastases would undergo surgery.

\section{Surgical Procedure}

In our hospital, 3 procedures were mainly conducted. The 3 -incision McKeown procedure was usually performed for tumors in the upper location, and the 2-incision Ivor-Lewis procedure and single-incision Sweet approach were always performed for tumors in the middle and lower locations. However, the choice of approach also depended on surgeon

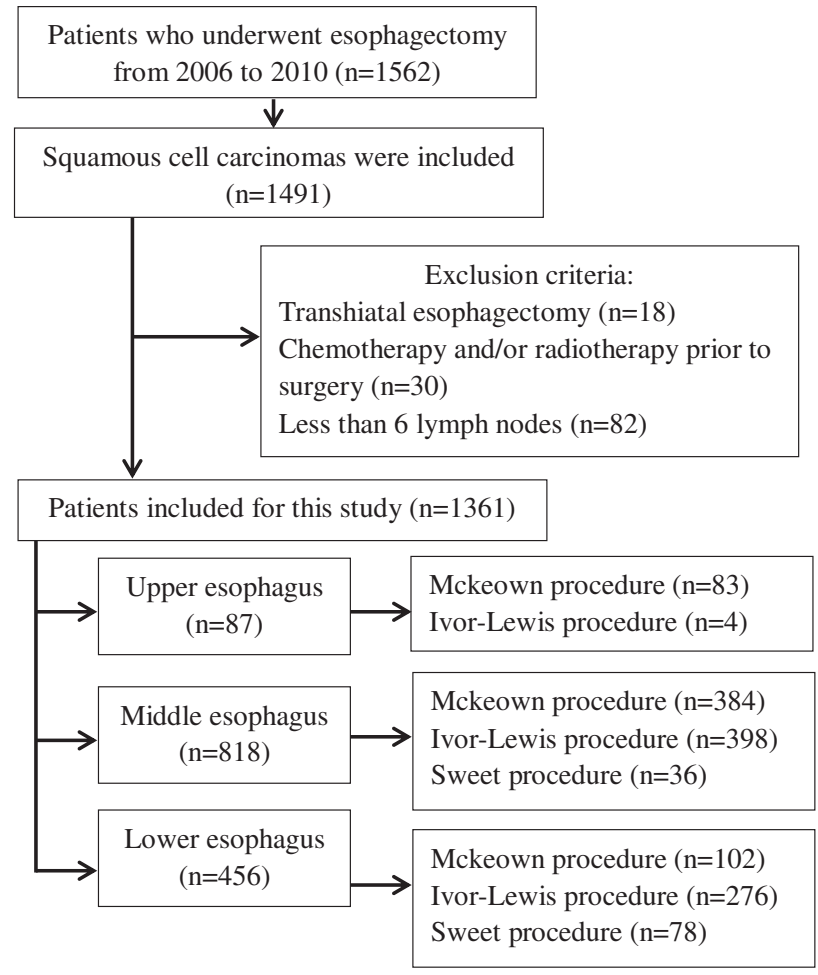

FIGURE 1. Patient disposition chart.
TABLE 1. Patient characteristics $(n=1361)$

\begin{tabular}{lc}
\hline \multicolumn{1}{c}{ Characteristic } & Patients (n) \\
\hline Age (y) & \\
$\leq 60$ & $749(55.0)$ \\
$>60$ & $612(45.0)$ \\
Gender & \\
Male & $1107(81.3)$ \\
Female & $254(18.7)$ \\
Tumor length (cm) & \\
25th percentile & 2.5 \\
50 th percentile & 3.5 \\
75th percentile & 4.5 \\
T classification & \\
Tis & $12(0.9)$ \\
T1 & $185(13.6)$ \\
T2 & $353(25.9)$ \\
T3 & $772(56.7)$ \\
T4a & $39(2.9)$ \\
N classification & \\
N0 & $647(47.5)$ \\
N1 & $396(29.1)$ \\
N2 & $217(15.9)$ \\
N3 & $101(7.4)$ \\
Lymphovascular invasion & \\
Yes & $266(19.5)$ \\
No & $1095(80.5)$ \\
Tumor differentiation & \\
Well & $132(9.7)$ \\
Moderate & $903(66.3)$ \\
Poor & $326(24)$ \\
\hline
\end{tabular}

Data in parentheses are percentages.

preference. In the right thoracic procedures (McKeown and Ivor-Lewis procedures), a muscle-sparing incision was made in the fourth intercostal space, the azygos vein was ligated and dissected, the esophagus was mobilized and dissected from the apex of the chest to diaphragmatic level, and thoracic duct was routinely ligated above the diaphragm. During mobilization of the stomach, the left gastric vein and artery were isolated and closed, and the right gastroepiploic vessels were carefully preserved. A gastric tube, about $4 \mathrm{~cm}$ in width, was completed along the greater curvature. In the McKeown procedure, the gastric tube was drawn to the left neck through the retrosternal route or posterior mediastinal route, and an end-to-end esophagogastric anastomosis was completed with manual suturing. In the Ivor-Lewis procedure, the gastric tube was pulled into the chest, and an end-to-side circular stapled anastomosis was made. In the left thoracic procedure (Sweet procedure), an incision through the sixth or seventh intercostal space was performed, the diaphragm was incised to gain sufficient exposure for the operation, and an end-to-side esophagogastric anastomosis was completed in the left chest with a circular stapler. In the present study, the gastric conduit was used as reconstruction substitute in all the patients.

\section{Lymphadenectomy}

Total mediastinal lymphadenectomy was routinely performed through a right thoracotomy (McKeown and Ivor-Lewis procedures), and through a left thoracotomy, usually the middle and lower mediastinal nodes and upper abdominal nodes were removed. When lymphatic involvement in the neck was indicated by CT scan or ultrasonography, cervical lymphadenectomy was performed through a collar incision (Table 2). The resected lymph nodes were labeled for pathologic examination according to the anatomic sites. In the present study, the cervical lymph nodes included the 
TABLE 2. Extent of lymphadenectomy and number of lymph nodes sampled

\begin{tabular}{llcc}
\hline & & & 2-FL \\
\cline { 3 - 4 } \multicolumn{1}{c}{ Variable } & 3-FL $(\mathbf{n}=\mathbf{2 9 4})$ & Right thoracotomy $(\mathbf{n}=\mathbf{9 5 3})$ & Left thoracotomy $(\mathbf{n}=\mathbf{1 1 4})$ \\
\hline Neck & $294(100.0), 9 / 14 / 21$ & & $40(35.1), 0 / 0 / 1$ \\
Upper mediastinum & $212(72.1), 0 / 2 / 5$ & $698(73.2), 0 / 2 / 5$ & $86(75.4), 1 / 4 / 9$ \\
Middle mediastinum & $266(90.5), 2 / 5 / 9$ & $855(89.7), 3 / 6 / 10$ & $99(86.8), 2 / 4 / 6$ \\
Lower mediastinum & $154(52.4), 0 / 1 / 2$ & $572(60.0), 0 / 1 / 3$ & $98(86.0), 3 / 7 / 12$ \\
Abdomen & $267(90.8), 4 / 8 / 11$ & $901(94.5), 5 / 8 / 13$ & $114(100), 12 / 18 / 26$ \\
Total & $294(100), 25 / 32 / 43$ & $953(100), 14 / 20 / 27$ & \\
\hline
\end{tabular}

Data presented as number of patients with lymph nodes sampled, with percentages in parentheses, followed by 25 th/50th/75th percentiles. 2-FL, 2-Field lymphadenectomy; 3-FL, 3 -field lymphadenectomy.

lymph nodes in the supraclavicular and cervical paraesophageal regions. The upper mediastinal nodes included the upper paraesophageal lymph nodes and bilateral paratracheal lymph nodes. The middle mediastinal nodes included the subcarinal, middle paraesophageal, and bilateral hilar lymph nodes. The lower mediastinal nodes included the lower paraesophageal, posterior mediastinal, and diaphragmatic lymph nodes. The upper abdominal nodes included the paracardial, lesser curvature, greater curvature, left gastric, common hepatic, splenic, and celiac lymph nodes.

\section{Statistical Analysis}

Statistical analysis was performed using the SPSS statistical software, version 16.0 (SPSS, Chicago, Ill). Categorical variables were summarized as the frequencies and percentages. For the variables significantly associated with lymphatic involvement, we then fit a multivariate logistic regression model (forward stepwise procedure) to identify the risk factors significant for LMNs. The results were considered significant for $P<.05$.

\section{RESULTS}

A total of 33,253 lymph nodes were removed (median, 23; 25th and 75th percentiles, 15 and 31). Of the 1361 patients, $714(52.5 \%)$ had 2634 positive lymph nodes.

\section{Pattern of LNMs}

No patient with stage Tis tumor was found to have LNM. As the depth of tumor invasion increased, both the frequency of LNMs and the number of positive lymph nodes increased (Figure 2). For stage T1 tumors, LNMs occurred in $1(2.2 \%)$ of 45 patients with tumor limited to the mucosa (stage T1a), but in $46(32.9 \%)$ of 140 patients with tumor infiltrating the submucosa (stageT1b).

Of all the LNMs, the paratracheal lymph nodes were the most frequently involved $(15.9 \%)$, followed by the middle paraesophageal (14\%) and paracardial (11.2\%) lymph nodes. A good relationship was seen between the tumor location and LNM regions. Cervical involvement was more frequent in patients with tumor in the upper location, and abdominal involvement was more frequent in patients with tumor in the lower location. LNMs in the upper mediastinum were common, regardless of the tumor location (Figure 3 and Table 3).

More LNMs developed in patients who had undergone 3 -FL $(69 \%)$. The reason might have been related to our selection bias. "Skipping metastasis," referring to a patient with cervical and/or abdominal involvement but no mediastinal metastasis, developed in 197 (27.6\%) of 714 patients with positive lymph nodes (63 patients had undergone 3-FL and 134, 2-FL; Table 4).

\section{Risk Factors for LNMs}

Male gender, deeper tumor invasion, greater tumor length, poor tumor differentiation, and lymphovascular invasion were associated with a greater occurrence of LNMs.

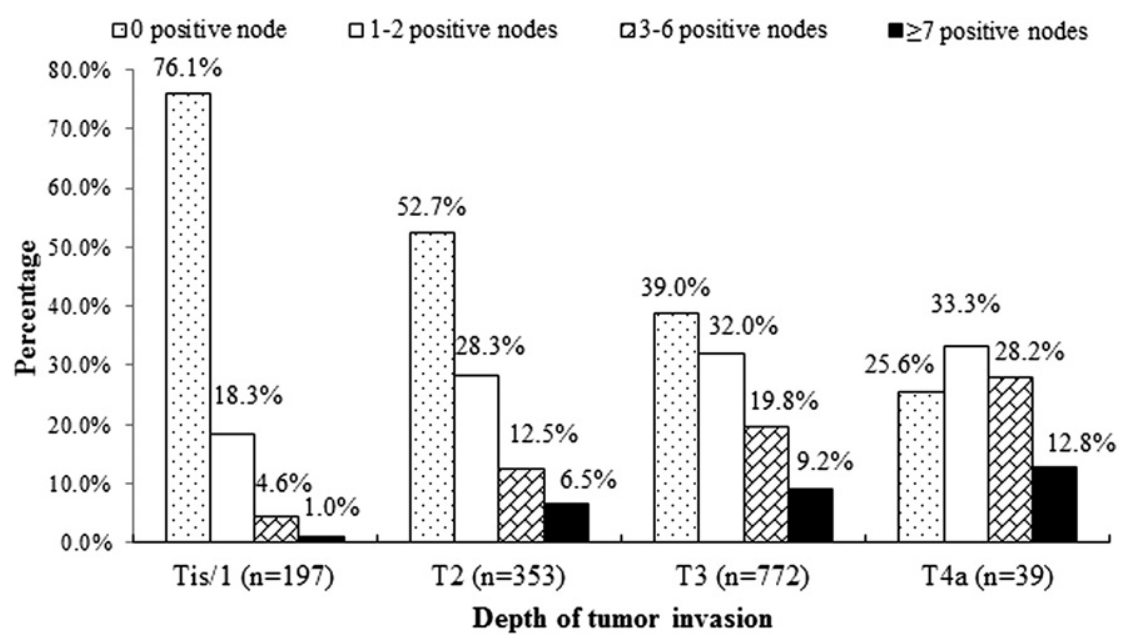

FIGURE 2. The frequency of lymph node metastases according to the depth of tumor invasion. 

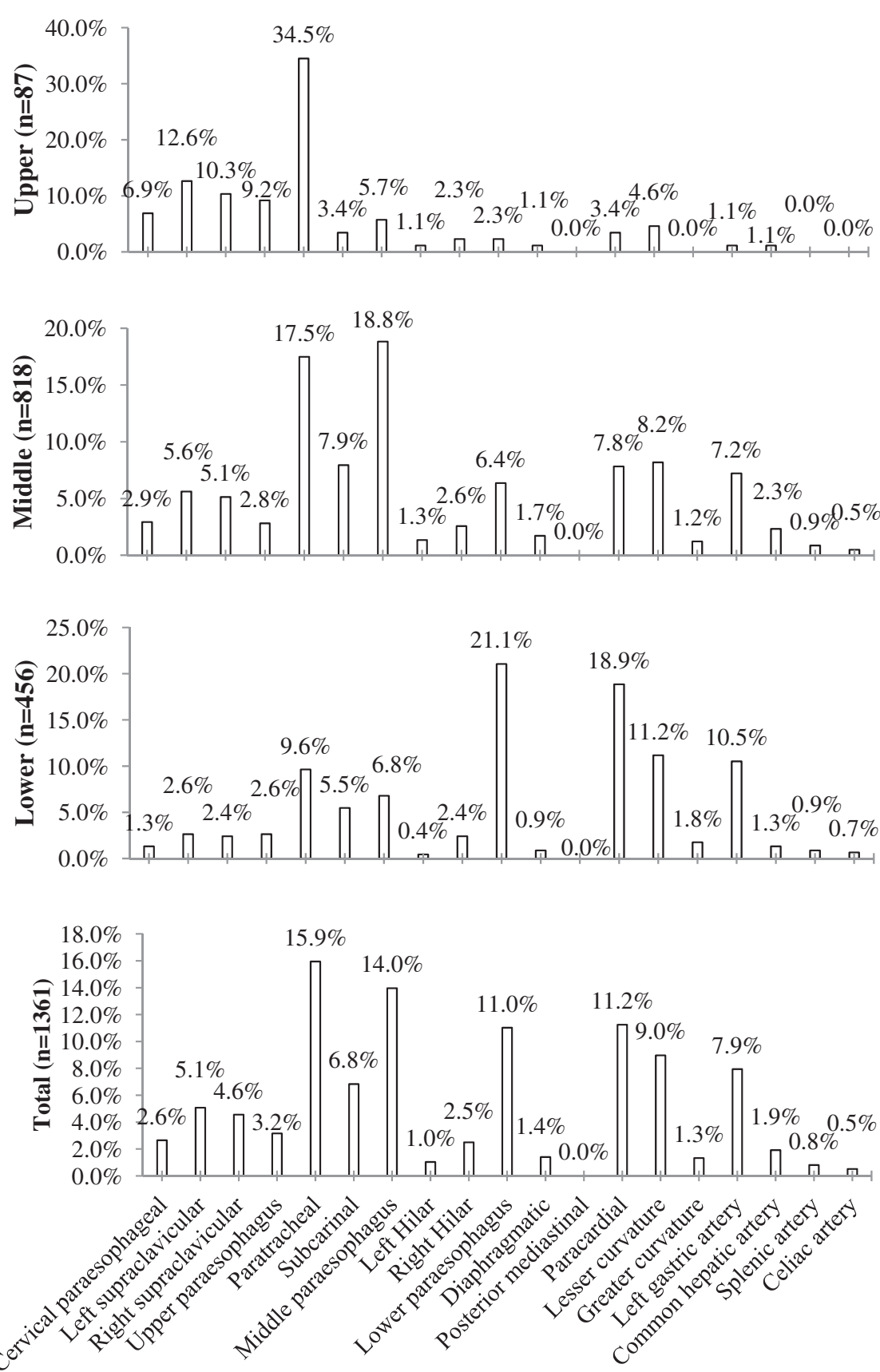

\section{Anatomic sites}

FIGURE 3. The frequency of lymph node metastasis according to the anatomic site.

In addition, cervical involvement was related to tumor location and paratracheal LNM (Table 5). Multivariate logistic regression analysis identified that greater tumor length $(P<.001)$, deeper tumor invasion $(P<.001)$, poor tumor differentiation $(P=.003)$, and lymphovascular invasion $(P<.001)$ correlated with the occurrence of LNMs (Table 6). In contrast, an upper tumor location $(P<.001)$, deeper tumor invasion $(P=.003)$, lymphovascular invasion $(P=.004)$, and paratracheal $\operatorname{LNM}(P=.002)$ were identified as risk factors for cervical involvement (Table 7).

\section{DISCUSSION}

Lymph node status, such as the number of positive nodes, number of fields with LNMs, and lymph node ratio, are among the most important predictors for survival after 
TABLE 3. Regions of lymph node metastases according to tumor location

\begin{tabular}{lcccc}
\hline \multirow{2}{*}{ Variable } & \multicolumn{4}{c}{ Tumor location } \\
\cline { 2 - 4 } & Upper $(\mathbf{n}=\mathbf{8 7})$ & Middle $(\mathbf{n}=\mathbf{8 1 8})$ & Lower $(\mathbf{n}=\mathbf{4 5 6})$ & Total $(\mathbf{n}=\mathbf{1 3 6 1})$ \\
\hline Neck & $21(24.1)$ & $87(10.6)$ & $25(5.5)$ & $133(9.8)$ \\
Upper mediastinum & $36(41.4)$ & $156(19.1)$ & $53(11.6)$ & $245(18.0)$ \\
Middle mediastinum & $9(10.3)$ & $197(24.1)$ & $51(11.2)$ & $257(18.9)$ \\
Lower mediastinum & $3(3.4)$ & $62(7.6)$ & $96(21.1)$ & $161(11.8)$ \\
Abdomen & $9(10.3)$ & $196(24.8)$ & $181(39.7)$ & $386(28.4)$ \\
\hline
\end{tabular}

Data presented as number of patients, with percentages in parentheses.

esophagectomy. ${ }^{7,8}$ However, it is still under debate about the extent of lymphadenectomy needed because of several factors, such as early lymphatic spread, complex distant metastasis, and unsatisfied prognosis after surgery. The present study determined the pattern of lymphatic spread by reviewing the data from all 1361 patients with ESCC who had undergone radical lymphadenectomy during the past 5 years at our hospital and the factors associated with LNMs. From these results, we hoped to provide useful information for guiding lymphadenectomy and future clinical trials.

LNM was found in $714(52.5 \%)$ of the 1361 patients and mostly occurred in the paratracheal, paraesophageal, and paracardial lymph nodes. Moreover, regarding the abdominal LNMs, we found the LNM mostly occurred in the perigastric areas, but common hepatic, splenic, and celiac LNMs were rare. These results confirmed the anatomic observations $^{9-11}$ that a long longitudinal extension of lymphatic drainage in the submucosa is connected to the superior mediastinum along the recurrent nerve and paracardial lymphatics. The lymph nodes in these sites should be carefully resected for tumor staging and to reduce local recurrence. In addition, good correlation was found between adjacent regional lymph node involvement and the tumor location. LNM was more frequent in the cervical area with tumors in the upper esophagus, but for middle and lower tumors, LNM was more frequent in the abdomen. We also found that LNMs in the upper mediastinum were common, regardless of the tumor location. On the basis of our data, we believe right thoracotomy with careful lymph node resection in the upper mediastinum should be conducted for curative intent.

Sentinel lymph node biopsy has become the reference standard for some superficial cancers; however, its use in ESCC is limited and controversial. ${ }^{12,13}$ Tumor cells can spread along the submucosal lymphatic channel for a long distance before entering the paraesophageal lymph nodes. Therefore, the presence of skip metastases was common in our patients with ESCC. A total of 197 patients (27.6\%) presented with skip metastases in the cranial and/or caudal directions without mediastinal LNMs. Cranial skip metastases occurred more frequently in patients who had undergone 3-FL, and caudal skip metastases were much more common overall. Thus, it would seem difficult to define the proper sentinel lymph node for ESCC.

The depth of tumor invasion was associated with LNMs and the number of positive lymph nodes. Once the tumor cells have breached the basement membrane of the epithelium, potential metastasis can develop. ${ }^{14}$ The cells spread from the mucosal lymphatic ducts to drain into a rich submucosal plexus and can then spread longitudinally through this dense lymphatic network and invade the paraesophageal lymphatic system. In addition to the deeper tumor invasion, a greater tumor length, poor tumor differentiation, and lymphovascular invasion were also identified as risk factors associated with LNM in our study. Our results

TABLE 4. Number of regions with lymph node metastasis

\begin{tabular}{|c|c|c|c|c|}
\hline Region (n) & Positive lymph node region & 3-FL $(n=294)$ & 2-FL $(n=1067)$ & Total $(n=1361)$ \\
\hline None & & $91(31.0)$ & $556(52.1)$ & $647(47.5)$ \\
\hline \multicolumn{5}{|l|}{1} \\
\hline & Neck & $38(12.9)$ & - & $38(2.8)$ \\
\hline & Mediastinum & $42(14.3)$ & $198(18.6)$ & $240(17.6)$ \\
\hline & Abdomen & $12(4.1)$ & $134(12.6)$ & $146(10.7)$ \\
\hline \multicolumn{5}{|l|}{2} \\
\hline & Mediastinum and neck & $47(16.0)$ & - & $47(3.5)$ \\
\hline & Abdomen and neck & $13(4.4)$ & - & $13(1.0)$ \\
\hline & Mediastinum and abdomen & $16(5.4)$ & $179(16.8)$ & $195(14.3)$ \\
\hline \multicolumn{5}{|l|}{3} \\
\hline & Neck, mediastinum, abdomen & $35(11.9)$ & - & $35(2.6)$ \\
\hline
\end{tabular}

Data presented as number of patients, with percentages in parentheses. 3-FL, 3-Field lymphadenectomy; 2- $F L$, 2-field lymphadenectomy. 
TABLE 5. Prevalence of lymph node metastases and cervical involvement according to patient and tumor characteristics

\begin{tabular}{|c|c|c|c|c|c|}
\hline \multirow[b]{2}{*}{ Characteristic } & \multirow[b]{2}{*}{ Patients (n) } & \multicolumn{2}{|c|}{ LNMs } & \multicolumn{2}{|c|}{ Cervical involvement } \\
\hline & & n $(\%)$ & $P$ value & n $(\%)$ & $P$ value \\
\hline Gender & & & $<.001$ & & .509 \\
\hline Male & 1107 & $608(54.9)$ & & $111(10.0)$ & \\
\hline Female & 254 & $106(41.7)$ & & $22(8.7)$ & \\
\hline Age (y) & & & .154 & & .740 \\
\hline$\leq 60$ & 749 & $406(54.2)$ & & $75(10.0)$ & \\
\hline$>60$ & 612 & $308(50.3)$ & & $58(9.5)$ & \\
\hline Tumor location & & & .284 & & $<.001$ \\
\hline Upper & 87 & $49(56.3)$ & & $21(24.1)$ & \\
\hline Middle & 818 & $415(50.7)$ & & $87(10.6)$ & \\
\hline Lower & 456 & $250(54.8)$ & & $25(5.5)$ & \\
\hline Tumor length $(\mathrm{cm})$ & & & $<.001$ & & .007 \\
\hline $0-0.9$ & 46 & $13(28.3)$ & & $1(2.2)$ & \\
\hline $1-1.9$ & 230 & $85(37.0)$ & & $15(6.5)$ & \\
\hline $2-2.9$ & 357 & $175(49.0)$ & & $28(7.8)$ & \\
\hline$\geq 3$ & 728 & $441(60.6)$ & & $89(12.2)$ & \\
\hline $\mathrm{T}$ classification & & & $<.001$ & & $<.001$ \\
\hline Tis-T1 & 197 & $47(23.9)$ & & $4(2.0)$ & \\
\hline $\mathrm{T} 2$ & 353 & $167(47.3)$ & & $29(8.2)$ & \\
\hline $\mathrm{T} 3$ & 772 & $471(61.0)$ & & 92 (11.9) & \\
\hline T4a & 39 & $29(74.4)$ & & $8(20.5)$ & \\
\hline Tumor differentiation & & & $<.001$ & & .386 \\
\hline Well & 132 & $42(31.8)$ & & $9(6.8)$ & \\
\hline Moderate & 903 & $476(52.7)$ & & $88(9.7)$ & \\
\hline Poor & 326 & $196(60.1)$ & & $36(11.0)$ & \\
\hline Lymphovascular invasion & & & $<.001$ & & $<.001$ \\
\hline Yes & 266 & $232(87.2)$ & & $45(16.9)$ & \\
\hline No & 1095 & $482(44.0)$ & & $88(8.0)$ & \\
\hline Paratracheal node involvement & & & & & $<.001$ \\
\hline Yes & 217 & & & $41(18.9)$ & \\
\hline No & 1144 & & & $92(8.0)$ & \\
\hline
\end{tabular}

LNM, Lymph node metastasis.

are consistent with those from previously published studies, ${ }^{15,16}$ and we believe all these factors should be considered when evaluating the lymph nodes status and therapeutic decision-making.

The International Society for Diseases of the Esophagus has classified the extent of lymphadenectomy as standard, extended, total, and 3-FL. ${ }^{17}$ However, the optimal extent

TABLE 6. Multivariate analysis of factors associated with lymph node metastasis

\begin{tabular}{|c|c|c|c|}
\hline & OR & 95\% CI & $P$ value \\
\hline $\begin{array}{l}\text { Tumor length } \\
\text { (per 1-cm increase) }\end{array}$ & 1.245 & $1.150-1.348$ & $<.001$ \\
\hline $\begin{array}{l}\text { Tumor invasion depth } \\
\text { (T3-T4/Tis-T2) }\end{array}$ & 1.719 & $1.343-2.201$ & $<.001$ \\
\hline $\begin{array}{l}\text { Tumor differentiation } \\
\text { (poor/well-moderate) }\end{array}$ & 1.509 & $1.145-1.988$ & .003 \\
\hline $\begin{array}{l}\text { Lymphovascular invasion } \\
\text { (yes/no) }\end{array}$ & 7.817 & 5.311-11.504 & $<.001$ \\
\hline
\end{tabular}

of lymphadenectomy remains controversial. In China, left thoracotomy has been widely performed; however, this procedure does not resect the lymph nodes in the upper mediastinum. From our retrospective analysis, and despite the long-term survival, we propose that extended total mediastinal lymphadenectomy through a right thoracotomy should be conducted. In the present study, in addition to the upper tumor location, deeper tumor invasion, and lymphovascular invasion, paratracheal lymph node involvement was identified as a risk factor for cervical lymph node metastases. This result is consistent with the findings from previous

TABLE 7. Multivariate analysis of factors associated with cervical lymph node metastasis

\begin{tabular}{lccr}
\hline & OR & $\mathbf{9 5} \%$ CI & $\boldsymbol{P}$ value \\
\hline Tumor location (upper/middle-lower) & 2.973 & $1.707-5.176$ & $<.001$ \\
Tumor invasion depth (T3-T4/Tis-T2) & 1.905 & $1.248-2.908$ & .003 \\
Lymphovascular invasion (yes/no) & 1.835 & $1.220-2.761$ & .004 \\
Paratracheal node involvement (yes/no) & 1.984 & $1.299-3.029$ & .002 \\
\hline$C I$, Confidence interval; $O R$, odds ratio. & & &
\end{tabular}


reports. ${ }^{18}$ Cervical lymphadenectomy or postoperative radiotherapy to the cervical region should be indicated for patients with positive paratracheal lymph nodes after 2-FL.

The pattern of lymphatic spread in our study differed from those of previous reports owing to different treatment strategies. The frequency of metastases was $9.8 \%, 18.0 \%$, $18.9 \%, 11.8 \%$, and $28.4 \%$ in the cervical, upper, middle, lower mediastinal lymph nodes and abdominal lymph nodes in the present retrospective study, respectively. The corresponding frequencies were $35.6 \%, 22.2 \%, 26.5 \%, 6.1 \%$, and $26.5 \%$ in 1850 patients with ESCC reported by Chen and colleagues ${ }^{4}$ and $3.8 \%, 5.0 \%, 28.6 \%, 16.5 \%$, and $21.6 \%$ in 1077 patients reported by Huang and colleagues. ${ }^{5}$ One major reason was the varying extent of lymphadenectomy. Because 3-FL was selectively conducted according to the $\mathrm{CT}$ and ultrasound findings in our center, we considered the patients who underwent 2-FL as undergoing radical surgery without cervical involvement and included all these patients in the present analysis to balance the selection bias. The other reason was the different patient cohorts. More superficial esophageal cancer (stage Tis-T1) was present in our research, and the proportion was $14.5 \%$ compared with about $5 \%$ in the other 2 studies.

\section{Strengths and Limitations}

The present study is one of the largest series to determine the pattern of LNMs in patients with thoracic ESCC. The database originated from a single, high-volume institute within a short period; thus, the data were more homogenous because the surgeons' practice patterns were more likely to be uniform. According to the pattern of lymphatic spread in the present study, we emphasized the importance of lymphadenectomy in the upper mediastinum. However, the present study had several limitations. First, it was a retrospective study, and the exact lymph node status could have been affected by the surgery types and surgeon preferences. Second, micrometastases in the lymph nodes of patients with stage $\mathrm{N} 0$ on routine histopathologic examination are reported to be frequent. ${ }^{19}$ However, we did not routinely examine this, although it had an influence on the real lymphatic status. Moreover, cervical lymphadenectomy was performed in selected patients according to our practice guidelines. Although cervical ultrasound examination was sufficient in the assessment of cervical nodal involvement with high sensitivity and specificity, ${ }^{20}$ the incidence of cervical metastases in the present study was underestimated owing to the image technology's limitations. The optimal extent of lymphadenectomy and the treatment strategy remain unanswered because of the lack of large prospective studies; thus, more research is still needed.

\section{CONCLUSIONS}

The mediastinum and abdomen are the predominant areas in which LNMs occurred more frequently in ESCC.
Extended total mediastinal and upper abdominal lymphadenectomy should be carefully performed. The depth of tumor invasion, lymphovascular invasion, and paratracheal lymph node involvement, together with the tumor location, might help us to conduct cervical lymphadenectomy individually in patients with ESCC.

\section{References}

1. Greenstein AJ, Litle VR, Swanson SJ, Divino CM, Packer S, Wisnivesky JP. Prognostic significance of the number of lymph node metastases in esophageal cancer. J Am Coll Surg. 2008;206:239-46.

2. Fujita H, Sueyoshi S, Tanaka T, Fujii T, Toh U, Mine T, et al. Optimal lymphadenectomy for squamous cell carcinoma in the thoracic esophagus: comparing the short- and long-term outcome among the four types of lymphadenectomy. World J Surg. 2003;27:571-9.

3. Tsurumaru M, Kajiyama Y, Udagawa H, Akiyama H. Outcomes of extended lymph node dissection for squamous cell carcinoma of the thoracic esophagus. Ann Thorac Cardiovasc Surg. 2001;7:325-9.

4. Chen J, Liu S, Pan J, Zheng X, Zhu K, Zhu J, et al. The pattern and prevalence of lymphatic spread in thoracic oesophageal squamous cell carcinoma. Eur J Cardiothorac Surg. 2009;36:480-6.

5. Huang W, Li B, Gong H, Yu J, Sun H, Zhou T, et al. Pattern of lymph node metastases and its implication in radiotherapeutic clinical target volume in patients with thoracic esophageal squamous cell carcinoma: a report of 1077 cases. $R a$ diother Oncol. 2010;95:229-33.

6. Edge SB, Byrd DR, Compton CC, Fritz AG, Greene FL, Trotti A. American Joint Committee on Cancer (AJCC) cancer staging manual. 7th ed. Chicago, Ill: Springer; 2010.

7. Shimada H, Okazumi S, Matsubara H, Nabeya Y, Shiratori T, Shimizu T, et al. Impact of the number and extent of positive lymph nodes in 200 patients with thoracic esophageal squamous cell carcinoma after three-field lymph node dissection. World J Surg. 2006;30:1441-9.

8. Liu YP, Ma L, Wang SJ, Chen YN, Wu GX, Han M, et al. Prognostic value of lymph node metastases and lymph node ratio in esophageal squamous cell carcinoma. Eur J Surg Oncol. 2010;36:155-9.

9. Kuge K, Murakami G, Mizobuchi S, Hata Y, Aikou T, Sasaguri S. Submucosal territory of the direct lymphatic drainage system to the thoracic duct in the human esophagus. J Thorac Cardiovasc Surg. 2003;125:1343-9.

10. Mizutani M, Murakami G, Nawata S, Hitrai I, Kimura W. Anatomy of right recurrent nerve node: why does early metastasis of esophageal cancer occur in it? Surg Radiol Anat. 2006;28:333-8.

11. Tachimori Y, Nagai Y, Kanamori N, Hokamura N, Igaki H. Pattern of lymph node metastases of esophageal squamous cell carcinoma based on the anatomical lymphatic drainage system. Dis Esophagus. 2011;24:33-8.

12. Grotenhuis BA, Wijnhoven BP, van Marion R, van Dekken H, Hop WC, Tilanus HW, et al. The sentinel node concept in adenocarcinomas of the distal esophagus and gastroesophageal junction. J Thorac Cardiovasc Surg. 2009; 138:608-12.

13. Thompson SK, Bartholomeusz D, Jamieson GG. Sentinel lymph node biopsy in esophageal cancer: should it be standard of care? J Gastrointest Surg. 2011;15: 1762-8.

14. Rice TW. Superficial oesophageal carcinoma: is there a need for three-field lymphadenectomy? Lancet. 1999;354:792-4.

15. Li H, Zhang Y, Cai H, Xiang J. Pattern of lymph node metastases in patients with squamous cell carcinoma of the thoracic esophagus who underwent three-field lymphadenectomy. Eur Surg Res. 2007;39:1-6.

16. Cen P, Hofstetter WL, Correa AM, Wu TT, Lee JH, Ross WA, et al. Lymphovascular invasion as a tool to further subclassify T1b esophageal adenocarcinoma. Cancer. 2008;112:1020-7.

17. Bumm R, Wong J. More of less surgery for esophageal cancer: extent of lymphadenectomy in esophagectomy for squamous cell esophageal carcinoma: how much is necessary? Dis Esoph. 1994;7:151-5.

18. Tabira Y, Yasunaga M, Tanaka M, Nakano K, Sakaguchi T, Nagamoto N, et al. Recurrent nerve nodal involvement is associated with cervical nodal metastasis in thoracic esophageal carcinoma. J Am Coll Surg. 2000;191: 232-7.

19. Laso CA, González JJ, Fresno F, Azcano E, Sanz L, Navarrete F. Prognostic value of micrometastases in esophageal and colorectal carcinoma (a clinical experience). Hepatogastroenterology. 2004;51:964-7. 
20. Cwik G, Dạbrowski A, Skoczylas T, Wallner G. The value of ultrasound in the assessment of cervical and abdominal lymph node metastases and selecting surgical strategy in patients with squamous cell carcinoma of the thoracic esophagus treated with neoadjuvant therapy. Adv Med Sci. 2011;56:291-8.

\section{Discussion}

Dr Antoon Lerut (Leuven, Belgium). Thank you, Dr Li, for this excellent presentation on an interesting topic, and thank you also for providing me with the manuscript well in advance.

In 1994, Dr Akiyama from Tokyo published in the Annals of Surgery his landmark report on radical lymph node dissection for cancer of the thoracic esophagus. His publication focused on a meticulous analysis of the pattern of lymph node spread in a series of 290 patients who all underwent 3-FL during a period spanning 20 years. Your presentation, now 20 years later, has the advantage that it included a very large cohort of 1361 patients within a short period of barely 5 years, making, of course, a much larger and more coherent group.

In the report by Akiyama, the incidence of cervical lymph node involvement was $38 \%$ overall, and for lower esophageal tumors, was as much as $27 \%$, indicating that, indeed, as you mentioned in your report, there is in your study material a bias in the sense that you performed 3 -FL in only $21 \%$ of your patients. Moreover, those were the patients who had on CT or ultrasound scan, a suspicion of positive lymph nodes in the neck.

I also would like to quote another publication, again from Japan, by Dr Nishimaki, on 3-FL, but this time specifically for early T1 tumors. In that report, $57 \%$ of the patients had positive nodes, of which as much as $16 \%$ were, again, in the cervical region.

Within this context, I have 3 questions. First, the purpose of your study was to obtain useful information to guide lymphadenectomy. Given the probably greater incidence of positive lymph nodes in the neck than you actually reported, would you consider performing systematic biopsy and frozen section analysis of the intrathoracic high paratracheal lymph node chain, and, in case of a positive report, would you then perform systematic cervical lymphadenectomy, in addition to the thoracic and abdominal compartments, irrespective of the tumor location and negative CT or ultrasound findings?

Dr Li. Thank you for your question, Professor Lerut.

This was a retrospective study, and we included all the patients who had undergone radical lymphadenectomy. Obviously, there was a selection bias in our study. At present, we cannot conduct cervical lymphadenectomy unselectively because of surgeon preference and in consideration of the high incidence of postoperative complications. Thus, the bias was present. However, it could be true that we could use the intraoperative information to select patients for cervical lymphadenectomy individually.

Dr Lerut. In the future?

Dr Li. Yes, and we will work on this aspect.

Dr Lerut. Thank you.

My second question-you did not speak on the number of involved lymph nodes. Is there a correlation between the number of positive intrathoracic lymph nodes and, again, the probability of having lymph node involvement in the cervical region? Furthermore, you showed that in $\mathrm{T} 1$ tumors you had an approximately $20 \%$ incidence of overall positive lymph node involvement. Did you have an opportunity to study the subdivision of the T1b tumors in submucosal area $(\mathrm{sm}) 1, \mathrm{sm} 2, \mathrm{sm} 3$ ? That correlates, of course, with the whole discussion on the indications for endoluminal therapies.

Dr Li. It is true a relationship exists between the number of positive lymph nodes in the mediastinum and metastasis in the cervical region in our analysis. Because of our selection bias for the patients who underwent 3-FL, more positive lymph nodes were usually found in the patients with an advanced tumor stage.

Regarding the subclassification of the T1b tumors, actually we only just finished this work last month. The proportion of superficial cancer was not large in our center, and it was about $15 \%$ in total. The incidence of metastases for sm1 and sm2 tumors was about $20 \%$, and it was about $38 \%$ for the sm 3 tumors.

Dr Lerut. So sm1 and sm2 was $20 \%$ overall?

Dr Li. Yes, it was about $20 \%$ for sm 1 and $\mathrm{sm} 2$ overall and about $30 \%$ for T1b tumors overall.

Dr Lerut. My third question is a more general question. The purpose of your study was also to obtain useful information for future clinical trials. In the United States and in Europe as well, most patients with the finding of 1 or more positive nodes on clinical staging currently will be treated with induction chemotherapy or chemoradiotherapy. From your report, it appears that only a small minority of patients received induction therapy. Thus, from your results and given the well-known negative prognostic effect of lymph node involvement, which was present in more than one half of your patients, are you considering to opt for induction therapy for patients who, on clinical staging, have positive nodes or do you believe that primary surgery with radical lymphadenectomy is to be preferred?

I would like to thank the Association for the privilege to discuss this highly interesting presentation.

Dr Li. In fact, induction therapy before surgery, or neoadjuvant chemotherapy or chemoradiotherapy, was not usually performed in our institution. Only 30 patients received chemotherapy or radiotherapy before surgery. However, in fact, what they received could not be regard as induction therapy or neoadjuvant therapy, because some of these patients underwent surgery owing to recurrence after radiotherapy, and some underwent surgery because they refused the chemotherapy after 1 or 2 cycles; they had changed their minds and requested surgery. Thus, we excluded these patients from the present study, and the proportion of these patients was small.

Dr Lerut. But would you in the future consider an increase in the indications for induction therapy?

Dr Li. Neoadjuvant therapy might be of benefit, and we will change our treatment strategy according to the findings of future clinical trials. But, at present, surgeons and most of the patients in our institution prefer surgery as the first choice.

Dr Lerut. Clever patients.

Dr Haiquan Chen (Shanghai, China). If I might answer the question, for squamous cell carcinoma, no data are available to show that neoadjuvant chemotherapy has a positive result. So, perhaps we will try that. We have a lot of patients. Perhaps we can initiate that trial.

Speaking of the sm1, sm2, and sm3, that is a new concept. We asked the pathologist to go back and review that in all the pathologic specimens. Our results on that study will come out soon. 
Dr Lerut. We are looking forward to that study next year.

Dr Chen. Sm1 and sm2, that was about 20\%, with no difference in $\mathrm{sm} 1, \mathrm{sm} 2$. However, $\mathrm{sm} 3$ was $38 \%$. That was high.

Dr Nasser K. Altorki (New York, NY). I enjoyed your presentation.

I just want to clarify something. When you speak about the paratracheal lymph nodes, that constitutes multiple stations of lymph nodes. What specific nodal stations did you actually dissect in the paratracheal area? Was that 2,3, or 4?

Dr Chen (Shanghai, China). Yes, there were several. Both bilateral recurrent nerves, specifically that level, bilateral recurrent nerves.

Dr Altorki. Do you dissect 2 or 4 ?
Dr Chen. For Ivor-Lewis, we do bilateral; the lymph nodes along the right recurrent nerve and left recurrent nerve were all removed.

Dr Altorki. Yes, but you did not dissect anything in front of the trachea.

Dr Chen. Yes, because metastasis in this region was rare according to our data, we did not routinely remove the lymph nodes anterior to the trachea.

Dr Altorki. I agree. I just wanted to make sure. I think it is important that when you state paratracheal that you actually specify the precise nodal station, because it could be commonly understood to include the nodes behind the cava and in front of the trachea.

Dr Chen. Yes, that is true. 